\title{
EZH2 is increased in paediatric T-cell acute lymphoblastic leukemia and is a suitable molecular target in combination treatment approaches
}

\author{
V. D'Angelo' ${ }^{1}$ A. lannotta ${ }^{1}$, M. Ramaglia ${ }^{1}$, A. Lombardi ${ }^{2}$, M. R. Zarone², V. Desiderio ${ }^{3}$, M. C. Affinita', G. Pecoraro ${ }^{1}$, \\ M. Di Martino ${ }^{1}$, P. Indolfi ${ }^{1}$, F. Casale ${ }^{1}$ and M. Caraglia ${ }^{2 *}$
}

\begin{abstract}
Background: T-cell Acute Lymphoblastic Leukemia (ALL) represents about 10-15\% of pediatric ALL cases. EZH2, one of the components of Polycomb group proteins (PRC2) complex, catalyzes the trimethylation of histone $\mathrm{H} 3$ lysine 27 that is associated with transcriptional repression and tumor development.

Methods: We examined the expression levels of PRC2 complex in primary samples of T cells ALL at diagnosis by western blotting and real time PCR. We evaluated the effect of 3-deazaneplanocin-A (DZNep), an EZH2 inhibitor, alone and in combination with Daunoblastine on cell viability, apoptotic death and cell cycle distribution of T cell established Jurkat cell line.

Results: EZH2 was expressed in $75 \%$ samples at different extents mainly with high expression level. SUZ12 was expressed in $60 \%$ samples and EED in all samples, respectively. The Kaplan-Meier analysis shows that T-ALL expressing EZH2 had a lower probability of disease-free survival (DFS) compared to T-ALL negative for EZH2 (23\% vs $100 \%)(p=0.01)$. The EZH2 inhibitor DZNep used in combination with Daunoblastine was synergistic in inducing growth inhibition and increasing the apoptosis in T-ALL Jurkat cells at 48 and $72 \mathrm{~h}$ paralleled by EZH2 decreased expression. Moreover, the combination decreased the activity of Erk-1/2 proliferation enzymes with no effects on Akt survival pathway.
\end{abstract}

Conclusions: The evaluation of EZH2 expression in pediatric T-ALL can be useful in predict the clinical outcome of the patients and EZH2 can be a useful target to improve the efficacy of conventional chemotherapy in this subset of patients with bad prognosis.

Keywords: EZH2, DZNep, T-cell Acute Lymphoblastic Leukemia

\section{Background}

Epigenetic alterations have an important role in leukemogenesis and Polycomb group proteins, which consist of two complexes (PRC1 and PRC2), represent a major class of epigenetic regulators in development and transcriptional repression. The polycomb repressive complexes (PRC) are key mediators of transcriptional repression and consists of three core subunits: EED

\footnotetext{
*Correspondence: michele.caraglia@unina2.it

${ }^{2}$ Department of Biochemistry, Biophysics and General Pathology, Second University of Naples, Via S.M. Costantinopoli, 16, 80138 Naples, Italy Full list of author information is available at the end of the article
}

(embryonic ectoderm development), SUZ12 (suppressor of zeste 12 homolog) and EZH2 (enhancer of zeste homolog 2). PRC2 controls the pivotal methylation of lysine 27 of histone H3 (H3K27) catalyzed by the SETdomain containing enhancer of zest homolog 2 (EZH2) protein and its cofactors [1]. Deregulation of this histone modification can lead to epigenetic silencing of genes that promote differentiation and leads to leukemogenesis [2]. Components of PRC2 are required for embryonic development and notably loss of EZH2 gene is associated with a block in B- and T-cell differentiation. Moreover, EZH2 acts as an oncogene, as it

\section{Ciomed Central}


is overexpressed in many solid cancers and lymphomas, in both advanced and metastatic diseases [3].

EZH2 is one of several chromatin regulatory proteins that have prompted great clinical and scientific interest as they offer the possibility of new therapeutic targets in cancer [4]. EZH2 contributes to cell fate decisions, orchestrating gene expression to control the balance between self-renewal and differentiation and has a predominant role in embryonic development becoming down regulated in some adult differentiated tissues [5].

T-cell acute lymphoblastic leukemia (T-ALL) represents about $10-15 \%$ of pediatric ALL cases and is generally associated with unfavorable clinical features and aggressive biologic behavior such as higher risk for primary resistant disease, early relapse and isolated central nervous system (CNS) relapse compared to B-progenitor ALL patients. The prognosis of T-ALL in children and adolescents has improved in recent years as a result of more intensive chemotherapy approaches but it remains worse compared to B-lineage ALL especially in presence of poor initial response to prednisone therapy [5].

Recent articles showed that EZH2 knockdown results in a significant decrease in cellular proliferation and invasiveness, suggesting that epigenetic therapy targeting PcG machinery to treat various tumors, and the development of drugs inhibiting the trimethylation of the lysine 27 on histone 3 (H3K27me3) can be effective therapy [6]. H3K27 methylation is catalyzed by the SET domain of EZH2 and is helped by 2 additional proteins, embryonic ectoderm development (EED) and suppressor of zeste 12 (SUZ12). These proteins, together with the histone binding proteins retinoblastoma binding protein 4 (RBBP4) and RBBP7, form the core components of PRC2 [7]. Other proteins such as PHD finger protein 1 (PHF1), which specifically stimulates H3K27 trimethylation rather than dimethylation sirtuin 1 (SIRT1), and jumonji, AT rich interactive domain 2 (Jarid2) are also found in PRC2 complexes likely acting as modulators [8].

3-deazaneplanocin-A (DZNep) is an interesting agent that blocks EZH2. DZNep is a promising tool for cancer therapy. It induces apoptosis of cancer cells but not of their normal counterparts: epithelial cells and fibroblasts [9] or more importantly hematopoietic cells [10]. The action of DZNep is due to its inhibition of S-adenosylhomocysteine hydrolase, which disrupts methionine metabolism and results in methyltrasferases inhibition [3]. Similarly to EZH2 knockdown, DZNep reverts epithelial-to-mesenchymal transition (EMT), and prevents tumor progression, making it a highly promising anti-tumour agent [6]. A key feature of epigenetic alterations in malignancy is their relative plasticity, unlike genetic mutations to DNA sequence that are definitive [4]. Hereafter, there have been increasing numbers of reports showing the impressive anticancer effects of
DZNep as a new epigenetic compound in a variety of cancer models both in vitro and in vivo [11-16]. The mechanisms and effects of DZNep have been studied in several solid tumors and acute myeloid leukemia, less is known about the potential of this compound for T-cell ALL [8].

Daunoblastine, a nonselective class I anthracycline, acts by binding to DNA-associated enzymes and intercalates the base pairs of the DNA's double helix. Although daunoblastine has been used as an anti-leukemic agent for decades, its success is often dependent on combination with other drugs [17].

In the present study, we examined the expression levels of EZH2, EED and SUZ12 in samples of T cells ALL. Moreover, we evaluated the effects of DZNep alone or in combination with Daunoblastine on established $\mathrm{T}$ cell Jurkat line.

\section{Methods}

Lymphoblastic leukemia cells were collected from pediatric patients diagnosed and treated for T-cell Acute Lymphoblastic Leukemia (T-ALL) at the Pediatric Oncology Unit of Second University of Naples and isolated from bone marrow at diagnosis with density gradient centrifugation Histopaque-1077 (1.077 g/ml; Sigma-Aldrich).

The study was approved by the Ethical Committee of the Azienda Universitaria Policlinico of the Second University of Naples (n. 94 on 21 January 2014) in compliance with the Helsinki Declaration. The informed consent for the participation to the study was approved and signed by the parents of the children.

\section{Protein extraction and western blot analysis}

Protein extraction was performed on ice for $30 \mathrm{~min}$ using lyses-buffer with protease-inhibitors. Total protein concentration was determined using Bradford assay (Bio-Rad). $30 \mu \mathrm{g}$ of total protein was run on $10 \%$ polyacrylammide gel and blotted onto PVDF membrane (Millipore, Marlborough, MA). Immunoblotting was performed using primary antibodies against EZH2 (C-1), EED (H-300), SUZ12 (D-10) Bcl2 (C-2) (Santacruz Biotechnology, INC). Primary antibodies AKT, pAKT, ERK, pERK were obtained from Cell Signaling. All secondary antibodies were obtained from Santa Cruz Biotecnology. All antibodies were used in accordance with manifacturer's instructions. Bands were visualized using a chemiluminescent system (ECL-Amersham). The intensity of each band was determined using a CCD camera and Quantity One 1-D analysis software (Biorad Laboratories). Results were normalized against the level of $\beta$ tubulin (Santa Cruz Biotechnologies) expression in each sample. It was obtained a range of expression of the bands from 0 to $175 \%$, with a median value of $60 \%$. Therefore, we have selected intensity values higher than $60 \%$ in order to consider the expression of the different 
proteins as high. Values of the intensities associated to the specific bands of the different proteins lower or equal to $60 \%$ were considered as low expression.

\section{RNA extraction and quantitative real-time PCR}

Total RNA was extracted from cell cultures using TRI REAGENT (Molecular Research Center Inc., OH, USA) according to the manufacturer's protocol. RNA from bone marrow at diagnosis was extracted with RNeasy FFPE kit (Invitrogen). The reactions were run on an ABI PRISM ${ }^{\circ} 7900$ HT Sequence Detection System; the cycling conditions were $10 \mathrm{~min}$ at $95{ }^{\circ} \mathrm{C}$ followed by 40 cycles of $15 \mathrm{~s}$ at $94{ }^{\circ} \mathrm{C}$ and $1 \mathrm{~min}$ at $68{ }^{\circ} \mathrm{C}$. In the first step, we determined the stability of a control gene ( $\beta$-actin) for the normalization of the real-time PCR products. Specific primers for human EZH2, SUZ12 and EED were designed (Table 1). Assays were performed in triplicate. We used the $2^{-\Delta \Delta C t}$ method to analyze the data obtained.

\section{Cell culture}

Human T cell leukemia, Jurkat cell lines, obtained from the German Collection of Microorganisms and Cell Cultures (DMSZ) were grown in RPMI media supplemented with heat inactivated $10 \%$ FBS and $1 \%$ Penicillin/ Streptomycin in a humidified atmosphere of $95 \%$ air/ $5 \% \mathrm{CO}_{2}$ at $37{ }^{\circ} \mathrm{C}$.

\section{Chemical reagents}

DZNep was purchased from Sigma-Aldrich, dissolved in distilled water and stored as frozen aliquots at $-20{ }^{\circ} \mathrm{C}$. Daunoblastine was obtained from Pfizer Pharma and diluted in physiological saline solution.

\section{Cell viability assay}

Cell viability was analyzed by MTT $[3-(4, \quad 5-$ dimethylthiazol-2-yl)-2, 5-diphenyl tetrazolium bromide] assay. Cells were seeded in 96-well plates at the density of 1 $\times 10^{3}$ cells/well in a final volume of $100 \mu \mathrm{L}$ and then incubated at $37{ }^{\circ} \mathrm{C}$ in a humidified atmosphere. Cells were treated with DZNep at a range of concentrations between 0.15 and $24 \mu \mathrm{M} / \mathrm{ml}$ and with Daunoblastine at a range of concentrations between 2.5 to $150 \mathrm{ng} / \mathrm{ml}$, in either single or combined treatments. Cells were exposed to a solution of $10 \%$ MTT to $48 \mathrm{~h}$ and $72 \mathrm{~h}$ from the treatments, for $4 \mathrm{~h}$ at

Table 1 Primer sequences for quantitative real time-polymerase chain reaction

\begin{tabular}{lll}
\hline Gene & Sense & Antisense \\
\hline EZH2 & 5'cgcttttctgtaggcgatgt 3' & 5'tgggtgttgcatgaaaagaa 3' \\
SUZ12 & 5'gggagactattcttgatgggaag 3' & 5'actgcaacgtaggtccctga 3' \\
EED & 5'gaaattccatccaagagatcca 3' $^{\prime}$ & 5'tggatattccataatcgtaaagca3' \\
B-actin & 5'gcgagaagatgacccagatc 3' $^{\prime}$ & 5'ggatagcacagcctggatag 3' \\
\hline
\end{tabular}

$37^{\circ} \mathrm{C}$ to form formazan crystals by reacting with metabolically active cells. The formazan crystals were solubilized in a $1 \mathrm{~N}$ isopropanol/HCL $10 \%$ solution at $37{ }^{\circ} \mathrm{C}$ for $30 \mathrm{~min}$. The absorbance was determined at $595 \mathrm{~nm}$ [18]. Cell viability was determined by the formula: Cell viability (\%) - (absorbance of the treated wells - absorbance of the blank control wells)/(absorbance of the negative control wells absorbance of the blank control wells) $\times 100 \%$. We have calculated 50 \% growth inhibition index (IC 50) for DZNep and Daunoblastine.

\section{Drug combination studies}

This study allowed the evaluation of the synergistic inhibition of cell growth produced by DZNep and Daunoblastine alone and in combination in Jurkat. Cells were seeded in 12-multi-weel plates at the density of $1 \times 10^{6}$ cells $/ \mathrm{ml}$ medium. After $24 \mathrm{~h}$ at $37{ }^{\circ} \mathrm{C}$ with $5 \% \mathrm{CO}_{2}$ in a humidified atmosphere, the cells were treated at the drugs range of concentrations described above. Drug combination studies were based on concentration-effect curves generated as a plot of the fraction of unaffected (surviving) cells versus drug concentration after $48 \mathrm{~h}$ of treatment. To explore the relative contribute of each agent to the synergism, the combination index (CI) for DZNep and Daunoblastine was obtained with software CalcuSyn. A CI of less than, equal to, and more than 1 indicates synergy, additivity, and antagonism, respectively as previously described [19].

\section{Cell cycle analysis}

Jurkat cell line were seeded at the density of $1 \times 10^{6}$ and treated with DZNep and Daunoblastine in humidified atmosphere at $37^{\circ} \mathrm{C}$ for $48 \mathrm{~h}$. After incubation, cells were washed in PBS, resuspended and directly stained in a Propidium Iodide (PI) solution for $30 \mathrm{~min}$ at $4{ }^{\circ} \mathrm{C}$ in the dark. Flow cytometry analysis was performed using FACScan (Becton Dickinson, San Jose, CA). To evaluate cell cycle, PI fluorescence was collected as FL2 (linear scale) by the ModFIT software (Becton Dickinson). For the evaluation of intracellular DNA content, at least 20,000 events for each point were analyzed in at least three different experiments giving a S.D. less than $5 \%$.

\section{Evaluation of apoptosis by DNA-flow cytometry}

Apoptotis was analyzed by Annexin-V kit (MedSystems Diagnostics, Vienna, Austria). During the early stages of apoptosis, phosphatidylserine residues translocated from the inner to the outer leaflet of the plasma membrane binding Annexin-V-FITC. Jurkat cell line was treated with DZNep and/or Daunoblastine, for $48 \mathrm{~h}$. After treatment, cells were collected and incubated with Annexin-V-FITC in a binding buffer (provided by the manufacturer) for 30 min at $4{ }^{\circ} \mathrm{C}$. Analysis of apoptotic cells was performed by flow cytometry (FACS Aria III, Becton Dickinson). For 
each sample, $10 \times 10^{4}$ events were acquired. Analysis was carried out by triplicate determination on at least three different experiments.

\section{AKT kinase assay}

Cells were cultured and treated with DZNep and/or Daunoblastine and lysed as previously described [20]. Then, $20 \mu \mathrm{l}$ of IgG1 anti-Akt monoclonal antibody agarose beads immobilized (Cell Signaling Technology) was added to $1 \mathrm{mg}$ of cell lysate and the mixture was incubated at $4{ }^{\circ} \mathrm{C}$ for the night in gentle agitation. The resulting immunoprecipitates were then incubated for $30 \mathrm{~min}$ at $30{ }^{\circ} \mathrm{C}$ with $1 \mathrm{mg}$ GSK-3 fusion protein (Cell Signaling Technology) in the presence of $200 \mathrm{mM}$ ATP and Kinase Buffer (25 mM Tris, pH 7.5, $5 \mathrm{mM}$ b-glycerophosphate, $2 \mathrm{mM}$ dithiotreitol, $0.1 \mathrm{mM}$ sodium orthovanadate, $10 \mathrm{mM}$ $\mathrm{MgCl} 2$ ). The reaction was terminated with the addition of $20 \mu \mathrm{l} 2 \times$ SDS sample buffer. The supernatants were boiled for $5 \mathrm{~min}$ and electrophoresed by $12 \%$ SDS-PAGE and the protein electro-transferred on a nitrocellulose film. Phosphorylation of GSK-3 was detected using as probe an anti-Phospho-GSK-3a/b (Ser21/9) rabbit polyclonal antibody (diluted 1:1000) and then with a secondary antirabbit HRP-conjugated monoclonal antibody, (diluted 1:2000). The film was washed with TBS $13-0.05 \%$ Tween 20 buffer and the specific reactivity was detected by chemiluminescence technique (Amersham) according to the manufacturer's instructions (Cell Signaling Technology).

\section{Statistical analysis}

PRC2 proteins and categories of different prognostic factors (gender, age, WBC count, relapse and prednisone response) was analyzed by chi-square test.

Then, the patients' dichotomous variables were assessed according to their influence on relapse and disease-free survival (DFS). DFS estimated at 5 years by the Kaplan-Meier analysis, was assessed using a log-rank test. In the DFS analysis, relapse and death in remission as a result of any cause were considered treatment failures. DFS was calculated for all patients that obtained complete remission from the date of remission to relapse. Results were expressed as probability (percent) and $95 \%$ confidence intervals $(\mathrm{CI})$. The hazard ratio (HR; with a $95 \% \mathrm{CI}$ ) for PRC2 subunits was estimated by the Cox regression analysis and categorized according to relapse risk prediction with the common prognostic factors. Data are given as mean \pm SD. Differences were assessed by $t$ test and $\mathrm{p}$ values $<0.05$ were considered statistically significant.

\section{Results}

Main clinical characteristics

Twenty patients (15 males and 5 females) were included in this study. The median age of the patients at diagnosis was 120 months. The whole group was enrolled in AIEOP-BFM treatment protocols. Morphologically the patients were stratified as L1 (4 cases) and L2 (16 cases), according to French American British (FAB) classification. At presentation, fourteen patients $(77.8 \%)$ had white blood cells (WBC) higher than $20,000 / \mathrm{mmc}$ and six $(22.2 \%)$ a lower count (Table 2). They were subdivided into three risk categories, according to their prognostic parameters: standard risk (SR) if a white cell count below 20,000/mmc, DNA index between 1.16 and 1.60 , good response to prednisone treatment (blasts reduction in peripheral blood $<1,000 / \mathrm{mmc}$ after 7 days treatment with steroids), no translocation $\mathrm{t}(9 ; 22)$ or $\mathrm{t}$ $(4 ; 11)$, complete remission at the conclusion of induction therapy; high risk (HR) if negative response to prednisone, evidence of translocation $\mathrm{t}(9 ; 22)$ o $\mathrm{t}(4 ; 11)$, not in remission at the conclusion of induction therapy, and Intermediate risk (IR) whose characteristics did not fall into either of the previous two categories. According to these features our patients were sub-classified as SR for 3 patients (15\%) and HR for 17 patients ( $85 \%)$. Clinical, pathological data and evolution of the 20 patients are listed in Table 2. Overall, with a median follow-up of 31 months (95 \% C.I 14.2-59.9), 11 patients (55\%) were alive and in continuous complete remission. Nine patients $(45 \%)$ relapsed.

Table 2 Clinical characteristics and pathologic data of 20 patients enrolled in the study

\begin{tabular}{ll}
\hline Clinical characteristics & Number of patients (\%) \\
\hline Gender & $15(75)$ \\
Male & $5(25)$ \\
Female & \\
WBC & $6(30)$ \\
$\leq 20.000 / \mathrm{mm}^{3}$ & $14(70)$ \\
$>20.000 / \mathrm{mm}^{3}$ & \\
FAB phenotype & $4(20)$ \\
L1 & $16(80)$ \\
L2 & \\
Disease Status & $9(45)$ \\
Relapsed & $11(55)$ \\
No relapsed & \\
Risk & $3(15)$ \\
Standard & $17(85)$ \\
High & \\
PDN & $7(35)$ \\
GR & $13(65)$ \\
PR &
\end{tabular}

WBC White Blood Cell, PDN Prednisone, GR Good Responders, $P R$ Poor Responders 
PRC2 expression in T-ALL primary patients

We have studied PRC2 expression and analysed the expression of mRNA in primary samples at diagnosis and on Jurkat cell line, by real time PCR based on RNA sample availability. EZH2 was markedly expressed like SUZ12 and EED (Fig. 1a); in details, the analysis showed from 0.5 to 7 fold higher expression of EZH2 compared to the control samples. We have also quantified protein expression by western blotting through the evaluation of the densities of the corresponding bands after laser scanning and representation as percentage. We have calculated the cut off values and we considered "low" expression values less than or equal to $60 \%$ and "high" expression higher than 60. EZH2 was expressed in 16/20 (75 \%) samples at different extents: 4 samples (25\%) showed low expression levels, whereas 12 samples (75 \%) had high levels. SUZ12 was expressed in 12 (60 \%) samples; 5 (41.6\%) showed low levels and 7 (58.3 \%) high expression. EED was expressed in all samples; 8 (40\%) had low expression and 12 (60\%) high expression (Fig. 1b-c). Moreover, Jurkat cell line expressed EZH2 and EED proteins only. When we compared EZH2, SUZ12 and EED expressions with

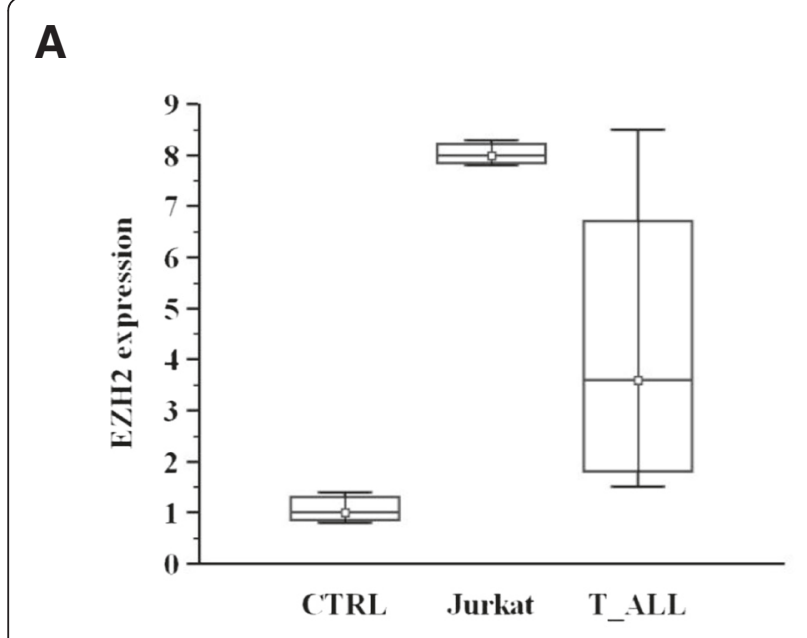

B

$\begin{array}{lllllllllll}\text { ALL } & 1 & 2 & 3 & 4 & 5 & 6 & 7 & 8 & 9 & 10\end{array}$

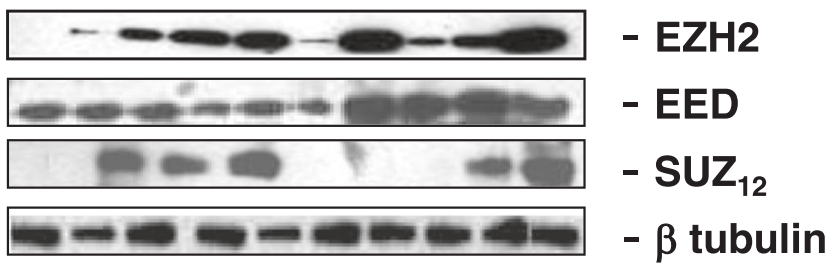

$\begin{array}{lllllllllll}\text { ALL } & 11 & 12 & 13 & 14 & 15 & 16 & 17 & 18 & 19 & 20\end{array}$

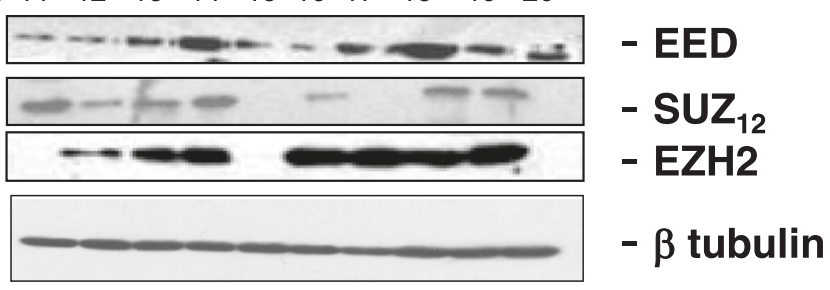

C

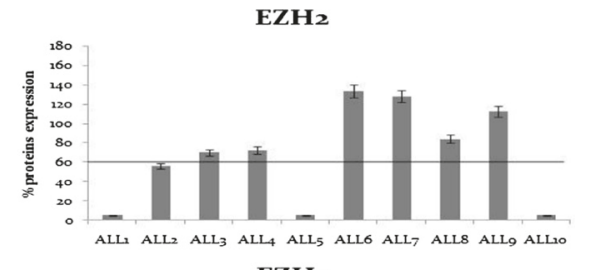

EZH2
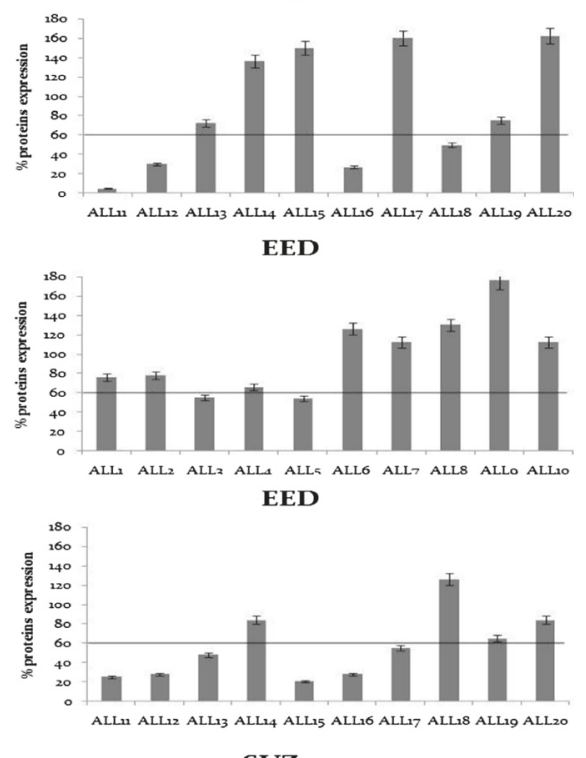

SUZ12

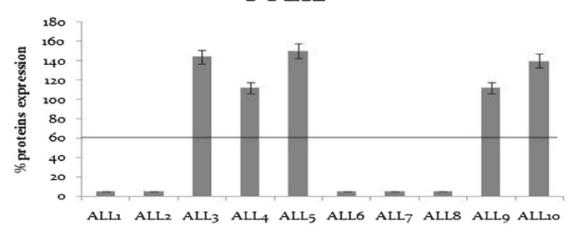

SUZ12

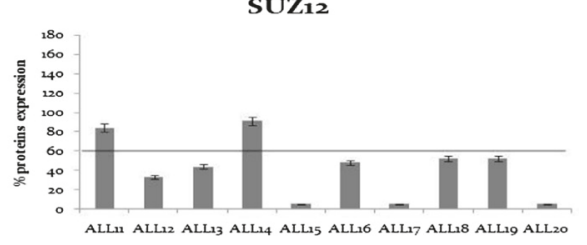

Fig. 1 EZH2 Expression (a) EZH2 expression evaluated through real time PCR in sample control (CTRL), Jurkat and T-ALL samples. PRC2 expression evaluated through immunoblotting (b) in T-ALL samples enrolled in the study and (c) representation of the densities of the bands corresponding to EZH2, SUZ12 and EED expressed as percentage of expression after laser scanning 
clinical and biological characteristics of patients, we did not found any significant correlation $(\mathrm{p}>0.05$, data not shown).

\section{EZH2, SUZ12 and EED expression and correlation with clinical outcome}

Estimated 5-year DFS by the Kaplan-Meier analysis shows that T-ALL expressing EZH2 had a lower probability of DFS compared to T-ALL negative cases for EZH2 (23\% vs $100 \%$ ) (log-rank test, p=0.02, Fig. 2a). These data were confirmed when negative and low expression samples were grouped vs high expression (22\% vs $100 \%$ ) (log-rank test, $\mathrm{p}=0.02$, Fig. 2b). Moreover, Kaplan-Meier analysis shows that patients with high EED expression had a lower probability of DFS vs low expression patients $(14 \%$ vs $86 \%)$ (log-rank test, $\mathrm{p}=$ 0.009 , Fig. 2c). No significant differences in DFS rate were found between patients stratified according to SUZ12 expression ( $43 \%$ vs $57 \%$ ) (log-rank test, $\mathrm{p}>0.05$, Fig. 2d). The Cox model, containing gender, age, initial
WBC count, PDN response, FAB phenotype and EZH2 expression suggests that EZH2 is not an independent prognostic factor $(\mathrm{p}>0.05)$.

\section{DZNep and Daunoblastine effects on Jurkat proliferation} Jurkat cell line is frequently used for delineating the mechanism of differential susceptibility of neoplasm for chemotherapy or radiation, which also emphasizes the significance of the line as a model for studies on T-acute lymphoblastic leukemia. DZNep and Daunoblastine reduced Jurkat number in a dose-dependent manner after 48 and $72 \mathrm{~h}$ from the beginning of incubation. DZNep induced $50 \%$ growth inhibition $\left(\mathrm{IC}_{50}\right)$ at 48 and $72 \mathrm{~h}$ with a concentration of $12 \mu \mathrm{M} / \mathrm{ml}$, respectively, while Daunoblastine induced a less pronounced growth inhibition reaching the $\mathrm{IC}_{50}$ after $72 \mathrm{~h}$ at a concentration of $12 \mathrm{ng} / \mathrm{ml}$. Moreover, only Daunoblastine was able to induce a time-dependent reduction of cell growth while DZNep reached a plateau after 48 h. (Fig. 3a-b)
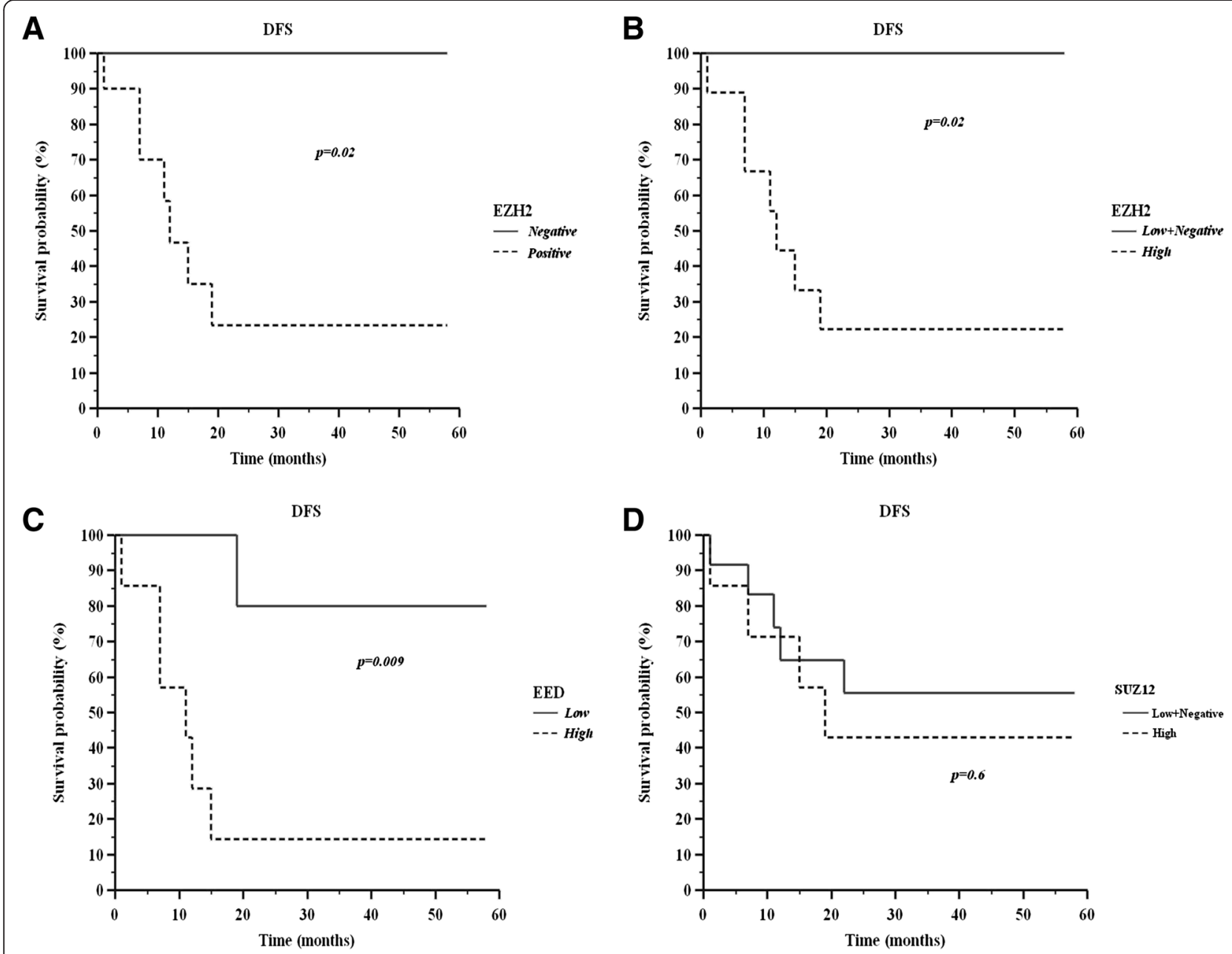

Fig. 2 Kaplan-Meier analysis. Disease-free survival (DFS) and EZH2 expression (a, b ), EED expression (c) and SUZ12 expression (d) 


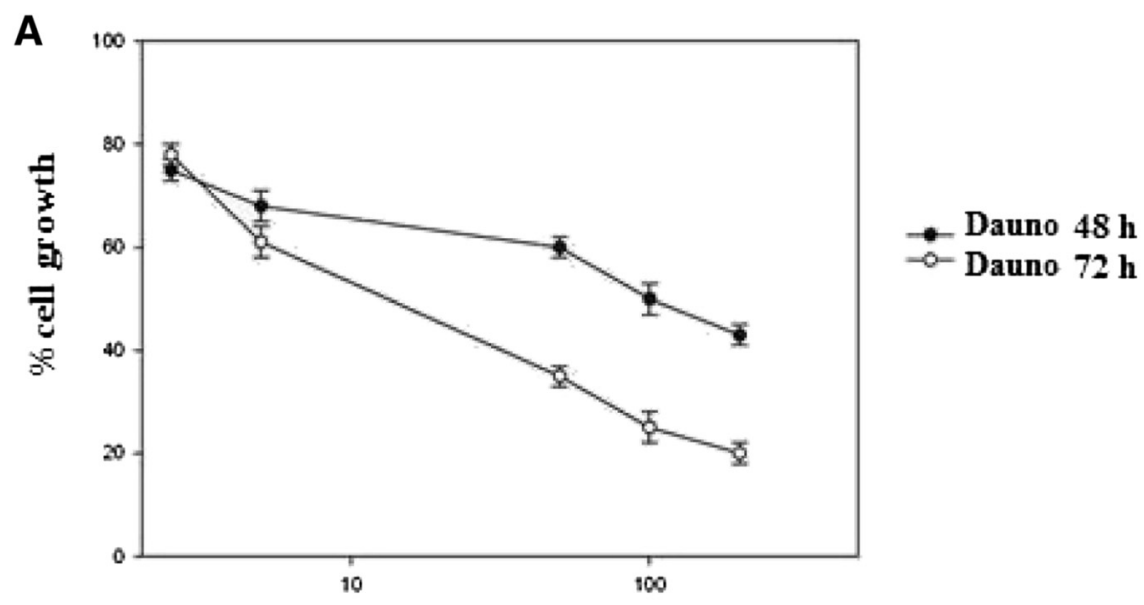

[] Concentration ng $/ \mathbf{m l}$

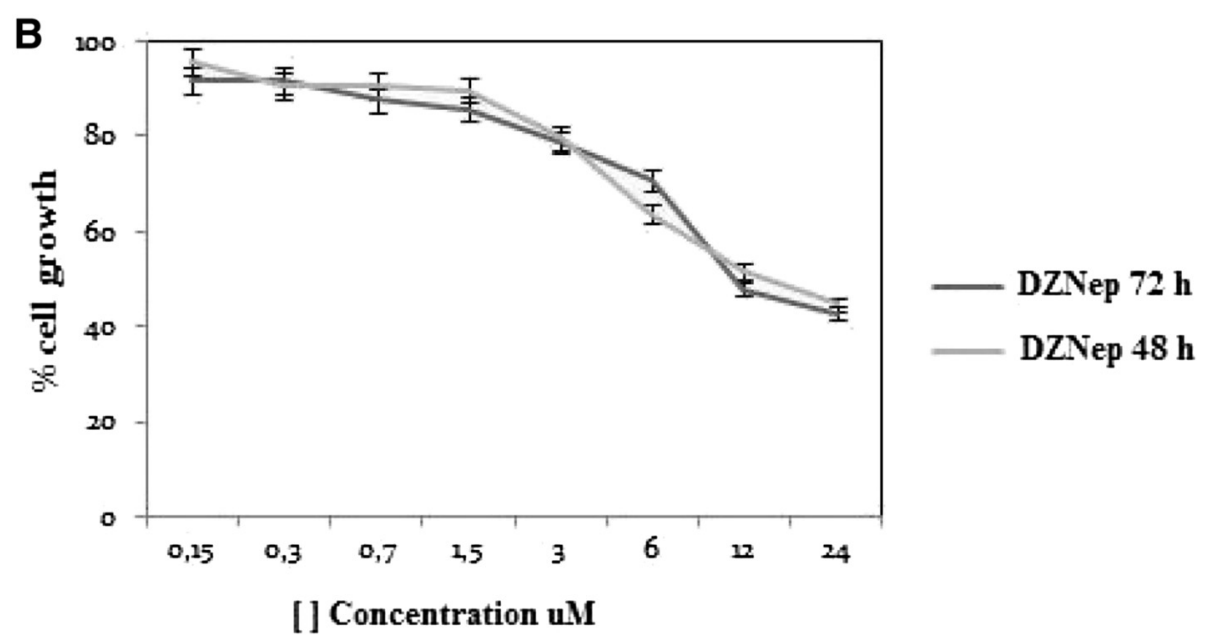

Fig. 3 DZNep and Daunoblastine in Jurkat cell growth. Dose-dependent inhibitory effect of Daunoblastine (a) and DZNep (b) on Jurkat cells treated with different concentrations of DZNep or Daunoblastine at 48 and $72 \mathrm{~h}$ as described in Materials and Methods section. Percentage of cell growth induced by different concentrations of agents was evaluated with MTT assay. Each point is the mean of three different evaluations performed in at least three different experiments

\section{DZNep synergizes with Daunoblastine in Jurkat cell growth inhibition}

We have evaluated the growth inhibition induced by DZNep in combination with Daunoblastine using data elaborated with the dedicated software Calcusyn (by Chou and Talalay, see also Materials and Methods section). The experimental $\mathrm{CI}_{50 \mathrm{~s}}$ (combination index calculated for $50 \%$ cell survival by isobologram analysis) values reveal that the DZNep/Daunoblastine combination was synergistic when both agents were used at (25:50 = Daunoblastine:DZNep) concentrations, in fact the $\mathrm{CI}_{50 \text { s }}$ were 0.6 at $48 \mathrm{~h}$ and 0.65 at $72 \mathrm{~h}$ (Fig. $4 \mathrm{a}-\mathrm{b}$ ).

These results suggested that the activity of a combination based on the use of an EZH2 inhibitor (DZNep) with a conventional chemotherapy agent (already used in high risk ALL at the clinical setting) could be useful in EZH2 over-expressing cells.

\section{Cell cycle analysis after DZNep in combination with Daunoblastine treatment}

We determined the effects of DZNep or Daunoblastine alone and DZNep/Daunoblastine treatment on Jurkat cell cycle. As demonstrated in Fig. 5, at 48 h, DZNep treatment increased the percentage of cells in S-phase from 25 to $60 \%$; DZNep used in combination with Daunoblastine determined an additional increase of cells in S-Phase reaching an about $70 \%$. At the same time, cells in $G_{0 / 1}$ phase decreased in a statistically significant manner $(\mathrm{p}<0.001)$. These results on cell-cycle distribution were confirmed at $72 \mathrm{~h}(\mathrm{p}<0.001)$. 


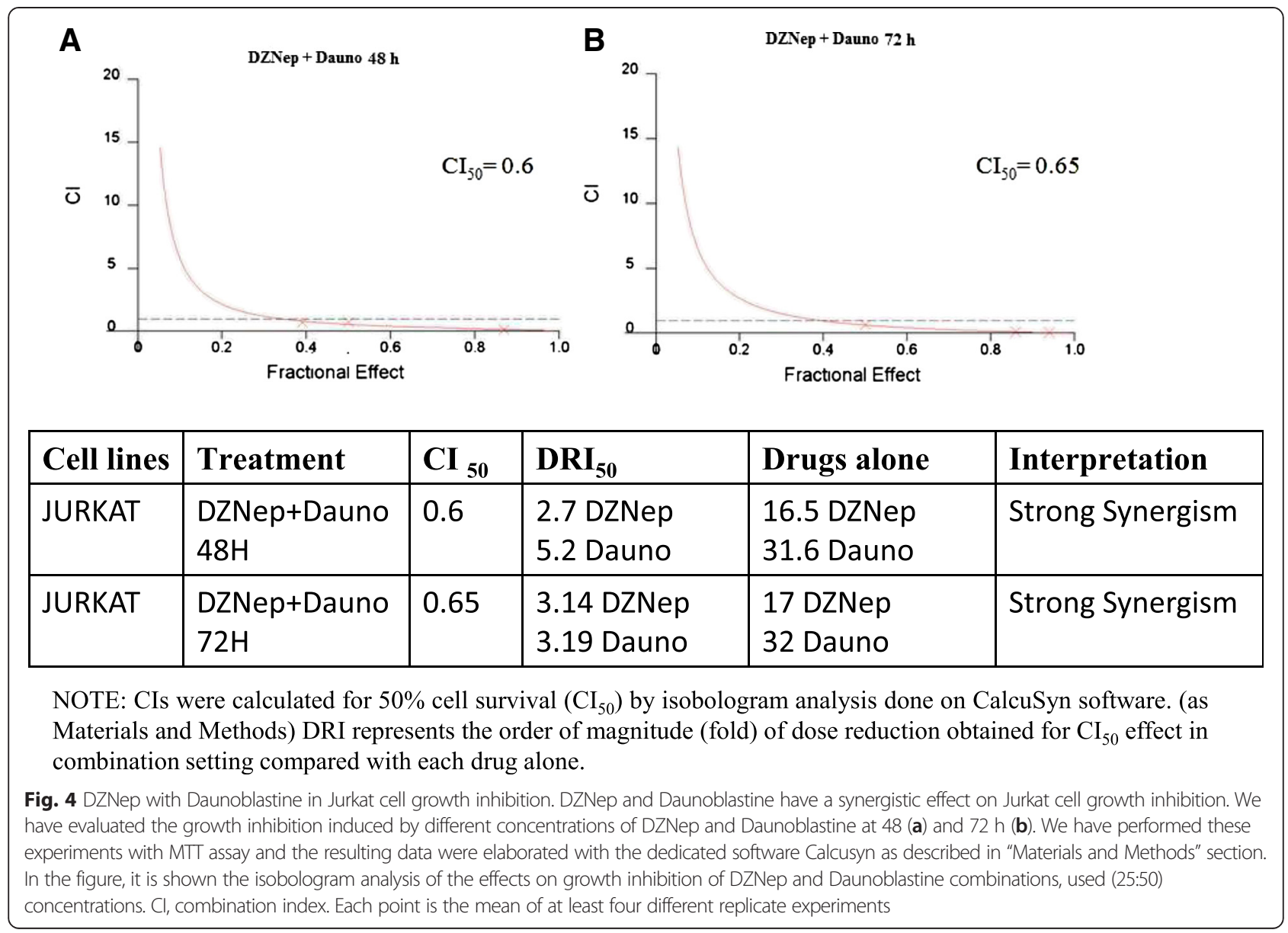

The synergistic effect of the DZNep/Daunoblastine combination on growth inhibition is paralleled by the apoptosis induction

After the treatment with DZNep and Daunoblastine, used at $\mathrm{IC}_{50}$ concentrations as described above, we have evaluated the induction of apoptosis on Jurkat by FACS analysis, after staining with Annexin V-FITC and PI, as described above. As shown in Fig. 6, we found an increase of apoptotic cells treated with the two agents in combination, compared to untreated cells or cells treated with the single agents. In details, we found that the treatment with DZNep for $48 \mathrm{~h}$ induced apoptosis in about $41.5 \%$ (34.6\% late and $6.9 \%$ early) of Jurkat cell population, while the treatment with Daunoblastine for $48 \mathrm{~h}$ induced apoptosis in about $27.9 \%$ (20.5 \% late and $7.4 \%$ early) $(\mathrm{p}<0.0001)$ of Jurkat population compared to $9.6 \%$ of untreated cells. On the other hand, when the cells were treated with DZNep + Daunoblastine, apoptosis was recorded in about $45.5 \%$ (34.3\% late and $11.2 \%$ early) of Jurkat cell population ( $\mathrm{p}<0.0001$ ) (Fig. 6a).

It is noteworthy that DZNep treatment for $72 \mathrm{~h}$ induced apoptosis in $63 \%$ (53.1 \% late and $9.9 \%$ early), Daunoblastine treatment in $32.1 \%$ (26.1 \% late and $6 \%$ early) and the combination in about $67 \%(52.2 \%$ late and $15 \%$ early) of Jurkat $(\mathrm{p}<0.0001)$ (Fig. 6b). On the basis of these findings, it can be assumed that the combination was able to induce strong apoptosis that was paralleled by the synergism on cell growth inhibition.

On the basis of these results, we have evaluated the effect of treatment with the agents alone or in combination on the mechanisms of apoptotic occurrence. The combination determined an increase of caspase- 3 and caspase- 9 cleaved isoforms, as a marker of their activity. Supporting the hypothesis of the activation in the apoptotic pathway, we observed a complete depletion of Bcl-2 in Jurkat cells treated with DZNep alone and in combination with Daunoblastine (Fig. 6c).

\section{DZNep treatment: $\mathrm{EZH} 2$ modulation in Jurkat}

We assessed EZH2 expression levels after DZNep and DZNep/Daunoblastine treatment in order to investigate upon the effects of the inhibitor in Jurkat cells. Quantitative RT-PCR analysis of EZH2 showed an about $50 \%$ decreased expression in cells treated with the single agents or the combinations at $72 \mathrm{~h}$ (Fig. 7a). Moreover, we found an almost complete EZH2 protein depletion 


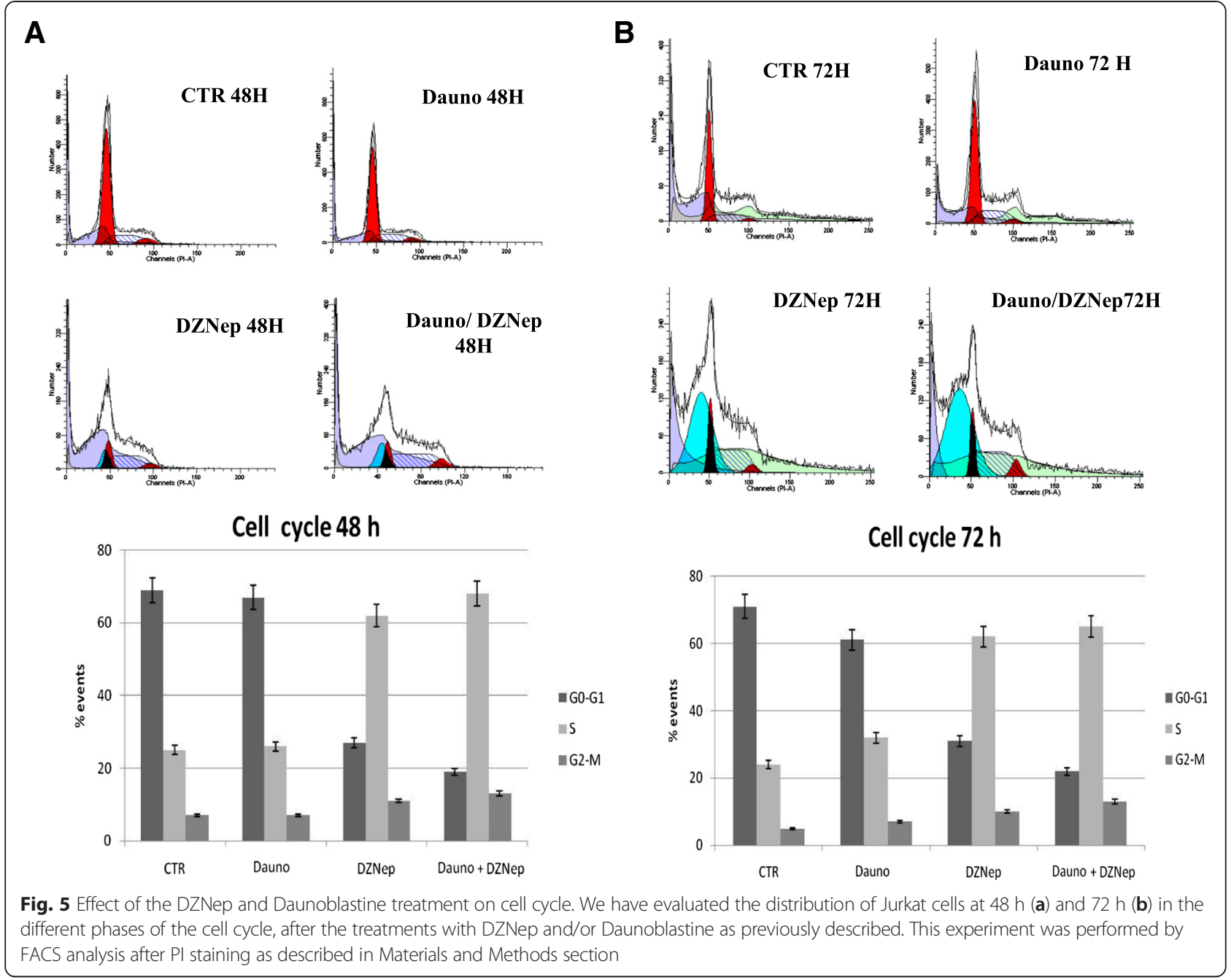

both in cells treated with DZNep and DZNep and Daunoblastine in combination at $48 \mathrm{~h}$ while an about $50 \%$ EZH2 protein reduction was recorded in Daunoblastinetreated cells (Fig. 7b).

\section{Effects of DZNep/Daunoblasatine combination on survival signal transduction}

Finally, we studied the effects of the agents on both the expression and activity of two key molecules involved in the regulation of both cell proliferation and survival processes. In details, to elucidate the signaling pathways underlying the effects of DZNep on Jurkat cells, MAPK (Erk-1/phosphorylated Erk-1/2) and AKT were examined.

In Jurkat cells, DZNep alone did not induce modulation in Erk-1/2 expression and activity while Daunoblastine caused an about $50 \%$ decrease of Erk-1/2 expression and phosphorylation if compared to untreated cells. The combined treatment strengthened the reduction of both activity and expression of Erk-1/2 (Figure 8 a, b and e).
We have found that DZNep alone and in combination did not induce significant changes in Akt expression. Moreover, we have evaluated the effect of the single agents and the combination on the modulation of Akt activity. In details, we found that neither the single agents nor the combination induced any significant changes on the activity of the survival Akt enzyme (Figure $8 \mathrm{c}$ and e).

\section{Discussion}

Stringent assessment of the relapse hazard in individual patients with ALL is an integral part of the modern approach to ALL therapy. Recently, the response to treatment is the most important prognostic factor in ALL. Indeed, many other variables have emerged as useful prognostic indicators. Moreover, the prognostic impact of age and, to a lesser extent, leukocyte count can be explained partly by their association with specific genetic abnormalities. It should be emphasized that primary genetic features do not entirely account for treatment outcome. For instance, up to 


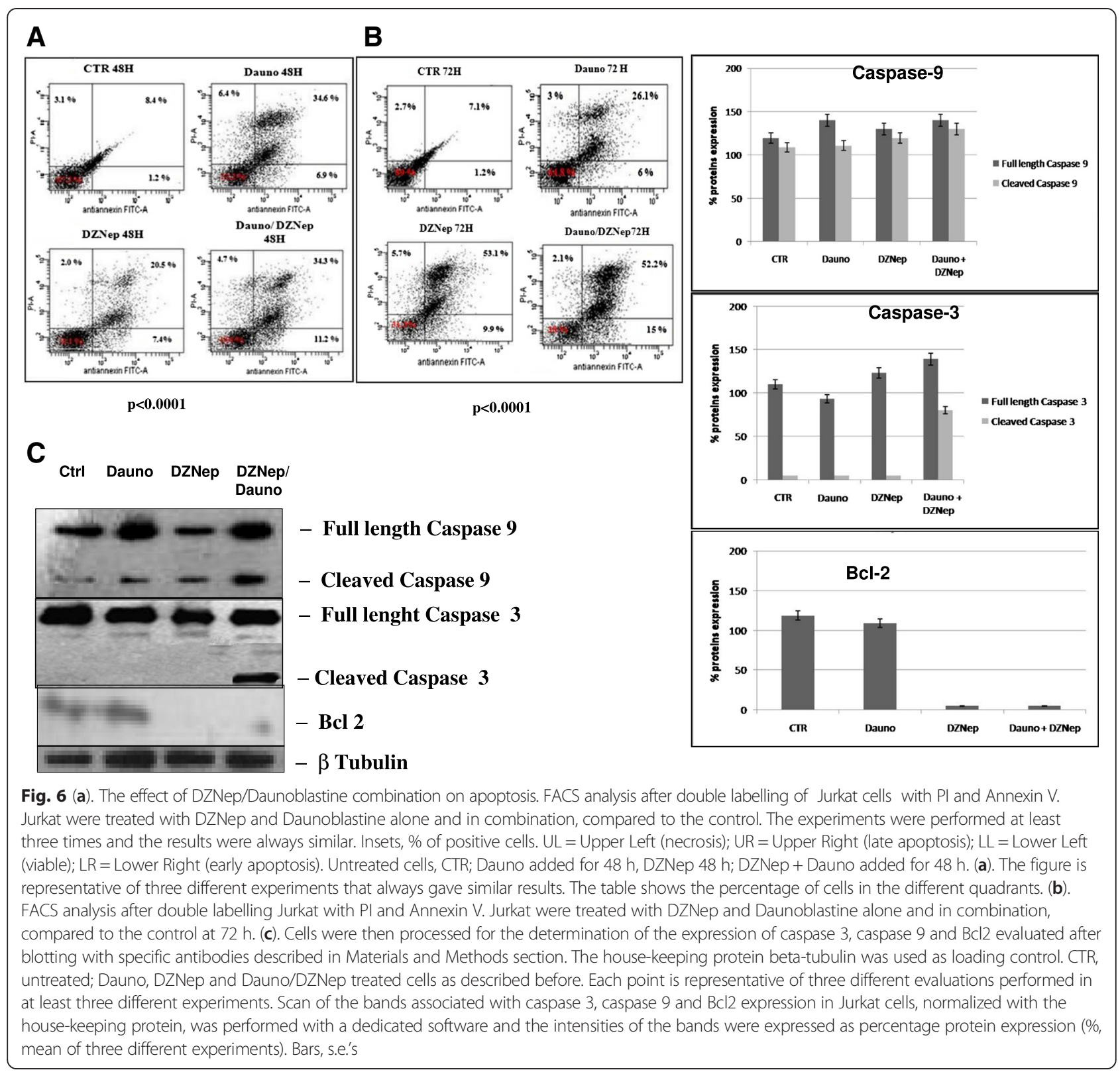

$20 \%$ of patients with favorable features as hyperdiploidy $>50$ chromosomes or TEL-AML1 fusion continue to suffer recurrences for their leukemia [21]. Moreover, inactivation of the p53 gene through mutations or overexpression of HDM2 whose product can bind to p53 and induce its degradation, is associated with a poor prognosis in children with ALL. The independent prognostic importance of a patient's gross early response to therapy has been recognized since the early 1980s. At the present, it is very important the measurement of minimal residual disease (MRD) by flow-cytometric detection of aberrant immunophenotypes or analysis by polymerase chain reaction (PCR) to afford a level of sensitivity and specificity that cannot be attained by traditional morphological assessment of treatment response [22].

In the last few decades, the survival in pediatric T-ALL patients has significantly improved, yet one patient in every four still encounters relapse and in most cases early. To date, few biomarkers have proved to be reliable in predicting T-ALL patient prognosis. The introduction of new factors that can lead to a more accurate patient risk stratification and the related treatment approach is expected to improve T-ALL outcome [22].

Recently, early T-cell precursor (ETP) ALL was identified as a T-ALL subgroup with a very poor outcome [23]. Moreover Zhang J et al. showed that ETP-ALL cases were characterized by activating mutations in genes 


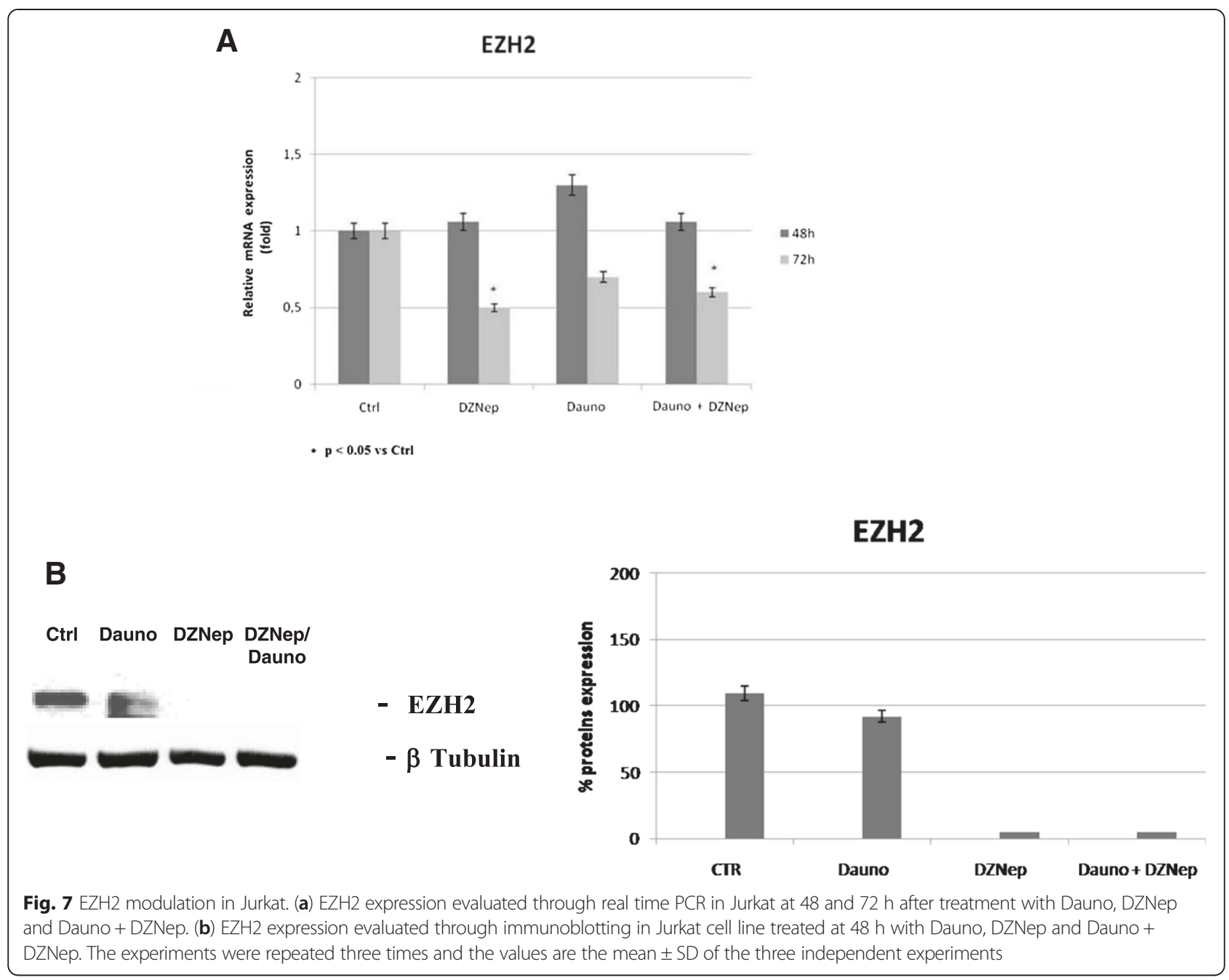

regulating cytokine receptor and RAS signaling, inactivating lesions disrupting haematopoietic development, and histone modifying genes (EZH2, EED, SUZ12, SETD2 and EP300) [24].

EZH2 overexpression is implicated in tumorigenesis and correlates to poor prognosis in several tumour types [25].

Moreover, EZH2 is considered one of the most appealing epigenetic targets for therapy in human cancer but efficiency of how it regulates its target genes depends on a number of additional factors, which may differ from one tumor cell line to another, even of the same tissue [26]. Here we have shown an expression of EZH2 and EED in 20 samples of T cells ALL and our findings suggest that their over-expression gives a lower probability of DFS. Therefore, EZH2 expression might be taken in account for a more precise and reliable patient stratification in order to improve treatment selection.

Epigenetic therapy in patients with hematological malignancies has produced interesting responses, but few long-term survivors [3]. Daunoblastine has been used as an anti-leukemic agent for decades, its success is often dependent on combination with other drugs. An interesting epigenetic agent that has the potential to increase anti-leukemic action of Daunoblastine could be DZNep. DZNep was first studied to enlarge the antiviral drugs arsenal and was further shown to induce cancer cell death [27]. DZNep became a promising anti-tumor drug with a significant efficacy on various cell types and no evident toxicity in vivo [28]. Studies by Momparler et al. revealed that sequential treatment of 5-AZA-CdR followed by DZNep showed a synergistic interaction with respect to its antileukemic activity as shown by a loss of clonogenicity for both human myeloid and murine lymphoid leukemic cell lines [3]. In this study, we demonstrated for the first time that DZNep and Daunoblastine used in combination at 25:50 concentrations, were synergistic at the Calcusyn elaboration. This suggested the opportunity to use EZH2 inhibitor and Daunoblastine combination in the treatment of paediatric T-ALL. Indeed, we observed that the synergistic combinations produced also a strong pro-apoptotic effect on Jurkat cell line. In details, DZNep alone and in combination with 


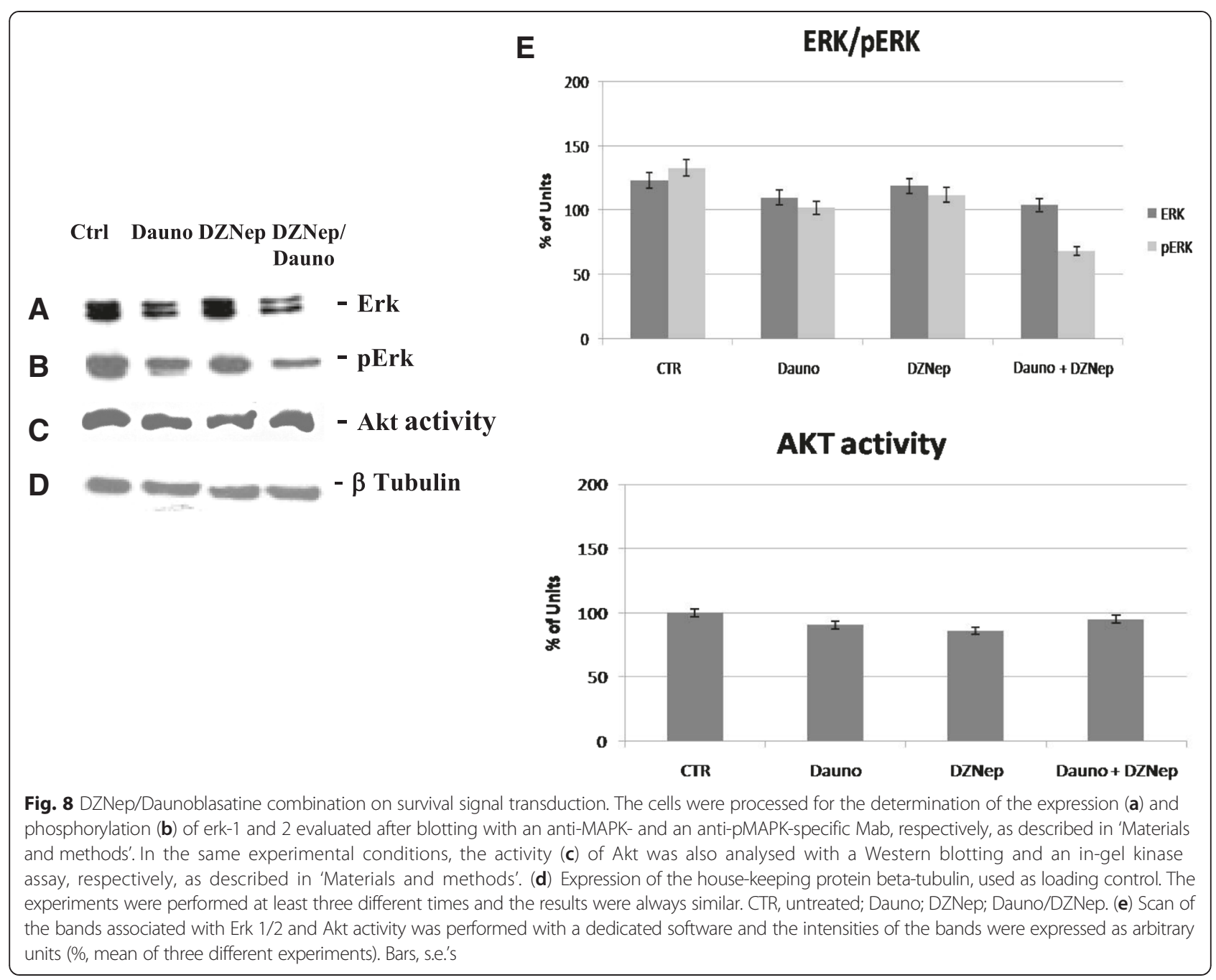

Daunoblastine induced $63 \%$ and $67 \%$ of apoptosis at $72 \mathrm{~h}$, respectively. It was shown that DZNep could inhibit the Jurkat cell proliferation activating probably checkpoints which cause cell cycle arrest at $S$ phase, resulting in acceleration of apoptosis. In fact, in natural killer/T-cell lymphoma, EZH2 upregulated via Myc-mediated mRNA inhibition, directly activates cyclin D transcription and promotes cell proliferation independently from methyltransferase activity [29]. Despite the main function of $\mathrm{EZH} 2$ is gene silencing through the methylation of H3K27, several studies have shown that EZH2 acts as trascriptional activator in various types of cancer independently from H3K27me. For example in natural killer/T-cell lymphoma, EZH2 upregulation directly activates cyclin D transcription via Myc-mediated mRNA inhibition and promotes cell proliferation independently from methyltransferase activity [29]. In the same way, EZH2 upregulation found in Jurkat cell line likely activates cyclin D transcription explaining the effect of DZNep obtained in our experimental system. Our results revealed that the treatment with both agents changed the expression and activity of many important proteins including caspase-3, 9, Bcl-2, Erk and EZH2. Among these proteins, the downregulation of Bcl-2 and up-regulation of cleaved Caspase3 and Caspase-9 are on-line with apoptosis occurrence observed in cells treated with the combination. At the same time, the combination was also able to modulate Erk-mediated proliferation pathway. In details, we observed that Erk phosphorylation was significantly inhibited by the combined treatment. This observation raises the possibility that treatment regimen with DZNep + Daunoblastine might be used to treat T-ALL cell, particularly the subgroup at worse prognosis.

\section{Conclusion}

Gene regulation by PRC2 complex is critical in tumorigenesis. Gene repression resulting from EZH2 activity is a key component of the epigenetic machinery with effects on both DNA and histone methylation. Our data on pediatric patients with T-ALL, confirm the involvement of 
EZH2 in the regulation of biological functions in T-ALL. Our study shows, for the first time, that reduction of EZH2 levels potentiates growth inhibition and apoptosis caused by Daunoblastine in Jurkat cell line. Pharmacological targeting of EZH2 might represent a potential feasible approach to be used as adjuvant treatment for making conventional therapy more effective in pediatric T-ALL.

\section{Competing interests}

The authors declare that they have no competing interests.

\section{Authors' contributions}

DAV and CM conceived of the study. LA and ZMR carried out the immunoassays. RM and IA performed the statistical analysis. PI and FC participated in the design of the study. DV, AMC, PG DMM, have made substantial contributions to conception the manuscript. All authors read and approved the final manuscript.

\section{Acknowledgements}

This work was supported in part by AlL (Italian Association Leukemia) and AGOP (Pediatric Oncology Association Parents). M.C. was supported by Regione Campania with a project entitled "Laboratori Pubblici Progetto Hauteville". The Jurkat cell line was acquired by "Smile to life "Voluntary Association Martina Santella.

\section{Author details}

'Department of Woman, Child and General and Specialized Surgery, Pediatric Oncology Unit - Second University of Naples, Via Luigi De Crecchio 4, 80138 Naples, Italy. ${ }^{2}$ Department of Biochemistry, Biophysics and General Pathology, Second University of Naples, Via S.M. Costantinopoli, 16, 80138 Naples, Italy. ${ }^{3}$ Department of Experimental Medicine, Section of Biotechnology and Medical Histology and Embryology, Second University of Naples, Naples, Italy.

Received: 28 May 2015 Accepted: 17 July 2015

Published online: 14 August 2015

\section{References}

1. Xie H, Xu J, Hsu Jessie H, Nguyen M, Fujiwara Y, Peng C, et al. Polycomb Repressive Complex 2 Regulates Normal Hematopoietic Stem Cell Function in a Developmental-Stage-Specific Manner. Cell Stem Cell. 2014;14:68-80

2. Momparler LR, Idaghdour Y, Marquez EV, Momparler FL. Synergistic antileukemic action of a combination of inhibitors of DNA methylation and histone methylation. Leukemia Research. 2012;36:1049-54.

3. Chase A, Cross NC. Aberrations of EZH2 in cancer. Cancer Res. 2011;9(17):2613-8.

4. Lund $\mathrm{K}$, Adams PD, Copland M. EZH2 in normal and malignant hematopoiesis. Leukemia. 2014;28:44-9.

5. Astolfi A, Vendemini F, Urbini M, Melchionda F, Masetti R, Franzoni M, et al. MYCN is a novel oncogenic target in pediatric T-cell acute lymphoblastic leukemia. Oncotarget. 2013;5:1.

6. Girard N, Bazille C, Lhuissier E, Benateau H, Llombart-Bosch A, Boumediene K, et al. 3-Deazaneplanocin A (DZNep), an Inhibitor of the Histone Methyltransferase EZH2, induces apoptosis and reduces cell migration in chondrosarcoma cells. PLOS One. 2014;9:5.

7. Sarma K, Marqueron $R$, Ivanov A, Pirrotta $V$, Reinberg D. Ezh2 requires PHF1 to efficiently catalyze $\mathrm{H} 3$ lysine 27 trimethylation in vivo. Mol Cell Biol. 2008;28:2718-31.

8. Zhou J, Bi C, Cheong LP, Mahara S, Liu SC, Koh T, et al. The histone methyltransferase inhibitor, DZNep, up-regulates TXNIP, increases ROS production, and targets leukemia cells in AML. Blood. 2011;18:10.

9. Fiskus W, Rao R, Balusu R, Ganguly S, Tao J, Sotomayor E, et al. Superior efficacy of a combined epigenetic therapy against human mantle cell lymphoma cells. Clin Cancer Res. 2012;18:6227-38.

10. Fiskus W, Wang $Y$, Sreekumar A, Buckley KM, Shi H, Jillella A, et al. Combined epigenetic therapy with the histone methyltransferase $\mathrm{EZH} 2$ inhibitor 3- deazaneplaocin A and the histone deacetylase inhibitor panobinostat against human AML cells. Blood. 2009;114:2733-43.

11. Puppe J, Drost R, Liu X, Joosse SA, Evers B, Cornelissen-Steijger $P$, et al. BRCA1-deficient mammary tumor cells are dependent on EZH2 expression and sensitive to Polycomb Repressive Complex 2-inhibitor 3-deazaneplanocin A. Breast Cancer Res. 2009;11:R63. doi:10.1186/bcr2354.

12. Crea F, Hurt EM, Mathews LA, Cabarcas SM, Sun L, Marquez VE, et al. Pharmacologic disruption of Polycomb Repressive Complex 2 inhibits tumorigenicity and tumor progression in prostate cancer. Mol Cancer. 2011;10:40. doi:10.1186/1476-4598-10-40.

13. Hayden A, Johnson PW, Packham G, Crabb SJ. S-adenosylhomocysteine hydrolase inhibition by 3-deazaneplanocin A analogues induces anti-cancer effects in breast cancer cell lines and synergy with both histone deacetylase and HER2 inhibition. Breast Cancer Res Treat. 2011;127:109-19.

14. Kikuchi J, Takashina T, Kinoshita I, Kikuchi E, Shimizu Y, Sakakibara-Konishi J, et al. Epigenetic therapy with 3-deazaneplanocin A, an inhibitor of the histone methyltransferase EZH2, inhibits growth of non-small cell lung cancer cells. Lung Cancer. 2012;78:138-43.

15. Zhaomei M, Hua L, Fernandez SV, Alpaugh KR, Zhang R, Cristofanilli M. EZH2 knockdown suppresses the growth and invasion of human inflammatory breast cancer cells. J Exp Clin Cancer Res. 2013;32:70.

16. Jiang X, Tan J, Li J, Kivimae S, Yang X, Zhuang L, et al. DACT3 is an epigenetic regulator of Wnt/beta-catenin signaling in colorectal cancer and is a therapeutic target of histone modifications. Cancer Cell. 2008;13:529-41.

17. Tacar $O$, Sriamornsak $P$, Dassa CR. Doxorubicin: an update on anticancer molecular action, toxicity and novel drug delivery systems. Journal of Pharmacy and Pharmacology. 2013;65:157-70.

18. Ilisso CP, Castellano M, Zappavigna S, Lombardi A, Vitale G, Dicitore A, et al. The methyl donor S-adenosylmethionine potentiates doxorubicin effects on apoptosis of hormone-dependent breast cancer cell lines. Endocrine. 2015;11.

19. Rossi M, Rotblat B, Ansell K, Amelio I, Caraglia M, Misso G, et al. High throughput screening for inhibitors of the HECT ubiquitin E3 ligase ITCH identifies antidepressant drugs as regulators of autophagy. Cell Death Dis. 2014;1:5:e1203.

20. Amodio N, Di Martino MT, Foresta U, Leone E, Lionetti M, Leotta M, et al. miR-29b sensitizes multiple myeloma cells to bortezomib-induced apoptosis through the activation of a feedback loop with the transcription factor Sp1. Cell Death Dis. 2012;29:3:e436.

21. Schrappe M, Hunger SP, Pui CH, Saha V, Gaynon PS, Baruchel A, et al. Outcomes after induction failure in childhood acute lymphoblastic leukemia. N Engl J Med. 2012;12(366):1371-81.

22. Milani G, Rebora P, Accordi B, Galla L, Bresolin S, Cazzaniga G, et al. Low PKCa expression within the MRD-HR stratum defines a new subgroup of childhood T-ALL with very poor outcome. Oncotarget. 2014;5:14.

23. Cousta- Smith E, Mullighan CG, Behm FG OM, Raimondi SC, Pei D, Cheng C, et al. Early T-cell precursor Leukemia: a subtype of very high risk acute lymphoblastic Leukemia. Lancet Oncol. 2009;10:147-56.

24. Pounds SB, Ulyanov A, Becksfort J, Gupta P, Huether R, Kriwacki RW, et al. The genetic basis of early T-cell precursor acute lymphoblastic leukemia. Nature. 2012;481:157-63.

25. Wang C, Liu Z, Woo CW, Li Z, Wang L, We JS, et al. EZH2 mediates epigenetic silencing of neuroblastoma suppressor genes CASZ1, CLU, RUNX3 and NGFR. Cancer Research. 2011. doi:10.1158/0008-5472.CAN-11-0961.

26. Ciarapica R, Miele L, Giordano A, Locatelli F, Rota R. Enhancer of zeste homolog $2(E Z H 2)$ in pediatric soft tissue sarcomas: first implications. BMC Medicine. 2011;9:63.

27. Tan J, Yang $X$, Zhuang L, Jiang $X$, Chen $W$, Lee $P L$, et al. Pharmacological disruption of Polycomb-repressive complex 2-mediated gene repression selectively induces apoptosis in cancer cells. Genes Dev. 2007;21:1050-10639.

28. Miranda T, Cortez CC, Yoo CB, Liang G, Abe M, Kelly TK, et al. DZNep is a global histone methylation inhibitor that reactivates developmental genes not silenced by DNA methylation. Mol Cancer Ther. 2009;8:1579-88.

29. Liu X, Chen X, Yu X, Tao Y, Bode AM, Dong Z, et al. Regulation of microRNAs by epigenetics and their interplay involved in cancer. J Experim Clin Cancer Res. 2013;32:96. 